\title{
A MULTIOBJECTIVE FACULTY-COURSE-TIME SLOT ASSIGNMENT PROBLEM WITH PREFERENCES
}

\author{
Nergiz A. ISMAYILOVA, Mujgan S. OZDEMIR, Rafail N. GASIMOV \\ n.ismail@ogu.edu.tr_mujgano@ogu.edu.tr gasimovr@ogu.edu.tr \\ Osmangazi University, Engineering and Architecture Faculty \\ Industrial Engineering Department, Bademlik 26030 \\ Eskişehir TURKEY
}

\begin{abstract}
A faculty-course-time slot assignment problem is studied. The multiobjective 0-1 linear programming model considering both the administration's and instructors' preferences is developed and a demonstrative example is included. Both modeling and solving such problems are difficult tasks due to the size, the varied nature, and conflicting objectives of the problems. The difficulty increases because the individuals involved in the problem may have different preferences related to the instructors, courses and time slots. The Analytic Hierarchy Process (AHP) and Analytic Network Process (ANP) are used to weight different and conflicting objectives. These weights are used in different scalarization approaches. The scalarized problems are solved using a standard optimization package, and solutions corresponding to AHP and ANP weights are compared.
\end{abstract}

Key words. Faculty course time slot problem, multi-objective optimization, conic scalarization, AHP.

\section{Introduction}

The problem of constructing timetables for educational institutions is a classical combinatorial problem that requires finding a schedule to determine which courses will be given in which classrooms by which instructors at which time slots. These problems, which are NP-complete mainly due to the associated constraints, have been studied in some detail over the last few decades among others by Even et al. (1976), de Werra (1985), Cooper and Kingston (1996), Daskalaki et al. (2004). In the academic environment there are organizational and individual constraints that influence the assignment problem. Due to the varied nature and the complexity of the problem, it is difficult to find a general procedure to solve such problems. In many cases it may be difficult to find even a feasible point. Therefore these problems have also been 
considered within different decomposion forms such as class teacher timetabling, faculty course and/or faculty course time slot assignments and so on.

Asratian and de Werra (2002) considered a theoretical model which extends the basic class teacher model of timetabling. This model corresponds to some situations which occur frequently in the basic training programs of university and schools. It has been shown that this problem is NP complete founded in some sufficient conditions for the existence of a timetable. Kara and Ozdemir (1997) presented a min max approach to the faculty course assignment problem by considering faculty preferences. Badri (1996) proposed a two-stage optimization model to maximize faculty-course preferences in assigning faculty members to courses and then faculty time preferences in allocating courses to time blocks. Badri's paper also describes an application of the model to the United Arab Emirates University. Hertz and Robert (1998) proposed an approach for tackling constrained course scheduling problem. Their main idea is to decompose the problem into a series of easier sub problems. Each sub problem is an assignment problem in which items have to be assigned to resources subject to some constraints. Daskalaki and Birbas (2005) also developed a two-stage relaxation procedure that solves the integer programming formulation of a university timetabling problem. Relaxation is performed in the first stage and concerns constraints that ensure consecutiveness in multi-period sessions of certain courses. These constraints, which are computationally more complex than the others, are recovered during the second stage and a number of sub-problems, one for each day of the week, are solved for local optima.

One of the main advantages provided by the decomposition of timetabling problems is that the solution process becomes easier than that of the whole problem. Compared to a solution approach that solves the problem in a single stage, computation time for decomposed problems is reduced significantly; nevertheless there may be some loss in the quality of the solution.

In this paper we consider a sub problem of the general timetabling problem in the form of faculty-course-time slot (FCT) assignments in a single stage. This study is a continuation and generalization of the faculty-course assignment problem considered earlier by 
Ozdemir and Gasimov (2004). They constructed a multi objective 0-1 nonlinear model for the problem, considering participants' average preferences and explained an effective way for its solution.

Note that the administration's and instructors' preferences in specific course and time slot assignments are important considerations. By considering these preferences, participants would be encouraged and as would thus also affect the student's performances during the lectures. As a result, the overall performance of the educational system is likely to increase. We develop a linear 0-1 multiobjective model for this problem in which objective functions related to the administration's total preferences on instructor-course and course-time slot assignments and instructors' total preferences on instructor-coursetime slot assignments would be maximized simultaneously. Besides, the model also includes the administration's objective functions to minimize the total deviation from the instructors' upper load limits. To demonstrate the features of our model a special example has been constructed. Because of the multiobjective nature of the FCT model, the solution process of this problem has been considered in two stages: scalarization of the given problem, and solving the scalarized problem. Because of the 0-1 nature of the problem the special scalarization approach called conic scalarization is applied. The Analytic Hierarchy Process (AHP) and the Analytic Network Process (ANP) are used to determine the weights of conflicting objectives. Efficient solutions corresponding to both sets of weights have been calculated and the results compared. GAMS/CPLEX solver was used to solve the scalarized problems.

Outline of the paper is as follows: The problem formulation and the corresponding mathematical model are presented in Section 2. Section 3 provides an illustrative example. Section 4 develops the solution approach, calculation of objective weights, scalarization and numerical results. Some conclusions drawn from the study are presented in Section 5.

\section{Problem formulation}

The model involves instructor-course-time slot assignments and instructor-course and course time slot assignments in a single stage. The term time slots used here is related to 
some partitioning of one teaching day. For example 8:00 am - 9:45 am, 10:00 am - 11:45 am, 1:00 pm - 2:45 pm, 3:00 pm - 4:45 pm and 5:00 pm - 6:45 pm may be a set of possible time slots. In this case some numeration can be assigned to time slots beginning at 8:00 am, 10:00 am, 1:00 pm, 3:00 pm and 5:00 pm as the $1^{\text {st }}$ time slot, the $2^{\text {nd }}$ time slot and so on, the $5^{\text {th }}$ time slot. It is assumed that each instructor is able to give more than one specific course. However each instructor may or may not be able to give all the courses considered.

The educational system (school) has the following kinds of requirements:

- Each course must be assigned to only one instructor.

- The weekly load of each instructor must be between his lower and upper limits.

- The number of parallel courses might be given on the same time slot is bounded by the availabilities (for example, by the number of available classrooms) of the school.

- The number of the same time slots assigned to each instructor is bounded. This requirement is made to prevent accumulation of all assignments of some instructor to an undesired (or to most desired) time slot. For example, all instructors may prefer time slot corresponding to 10:00 am - 11:45 am while a time slot corresponding to 5:00 pm - 6:45 pm may be undesirable.

The school administration has preferences for instructor-course and course-time slots assignments. Besides, the instructors have preferences for both courses that would be given by them and for time slots during which their assigned courses would be given. As we mentioned earlier, participants' preferences have an important role to increase the overall efficiency of the educational system. These preferences have been considered via objective functions in our model. The model parameters, decision variables and objective functions are defined as follows.

\section{Parameters}

Let

$I=\{i: i=1,2, \ldots, m\}$ be the set of instructors, $J=\{j: j=1,2, \ldots, n\}$ be the set of courses, 
$T=\{t: t=1,2, \ldots, s\}$ be the set of time slots,

$c_{t} \quad$ be the number of parallel courses might be given on time $t$,

$h_{j} \quad$ be the total number of lecture hours for $j$ th course in a week,

$l_{i}$ and $u_{i}$ be lower and upper hours of the $i$ th instructor's weekly load,

$a_{i j} \quad$ be the administration's preference level for the assignment of $j$ th course to the $i$ th instructor,

$b_{j t} \quad$ be the administration's preference level for the assignment of $j$ th course to the th the slot.

$p_{i j t} \quad$ be the preference level of $i$ th instructor for the $j$ th course to be given at time

slot $t$.

$d_{t} \quad$ be the maximum number for time slot $t$ that might be assigned to each instructor during the week.

$A \quad$ as a large positive constant, e.g. $A=S$.

$B \quad$ as a large positive constant, e.g. $B=m$.

\section{Decision variables:}

$y_{i j}= \begin{cases}1 & \text { if } i \text { th instructor is assigned to } j \text { th course } \\ 0, & \text { otherwise. }\end{cases}$

$z_{j t}= \begin{cases}1 & \text { if } j \text { th course is given on time } t \\ 0, & \text { otherwise. }\end{cases}$

$x_{i j t}= \begin{cases}1 & \text { if } j \text { th course is given by } i \text { th instructor on time } t \\ 0, & \text { otherwise. }\end{cases}$

Objective functions.

Objective functions are constructed by maximizing the preference levels of each instructor on instructor-course-time slot assignments and maximizing the administrations' total preference levels on instructor-course and course-time slot assignments. Maximization of preferences can be expressed as maximization or minimization of the corresponding objective function independently of the preference order defined. It can be defined for some scale for all preferences. For example the numbers $1,2, \ldots, 9$ can be 
assigned to preferences $a_{i j}\left(b_{j t}\right.$ and $\left.p_{i j t}\right)$, where the number 1 indicates the most desired case, and 9 the most undesired one. In this case the maximization of preferences will be expressed as minimization of the corresponding objective functions. Objective functions can be divided into two groups: objectives related to instructors' preferences on instructor-course-time slot assignments and objectives related to the administration's preferences on instructor-course, course-time slot assignments and an objective function related to the teaching load limits of instructors.

Let $(x, y, z)$ be a general vector of all decision variables: $x=\left(x_{i j t}, y_{i j}, z_{j t}\right), i \in I, j \in J, t \in T$.

We have the following objectives:

- Minimize the instructors' preference levels $L_{i}$ on course-time slot assignments:

$$
L_{i}(x, y, z)=\sum_{j \in J} \sum_{t \in T} x_{i j t} p_{i j t} h_{j}, i \in I .
$$

- Minimize the administrations total preference level on instructor-course assignments

$$
A_{1}(x, y, z)=\sum_{i \in I} \sum_{j \in J} a_{i j} y_{i j}
$$

- Minimize the administrations total preference level on course-time slot assignments

$$
A_{2}(x, y, z)=\sum_{j \in J} \sum_{t \in T} b_{j t} z_{j t}
$$

- Minimize the total deviation from the upper load limits of the instructors

$$
A_{3}(x, y, z)=\sum_{i \in I}\left(u_{i}-\sum_{j \in J} y_{i j} h_{j}\right)
$$

Formulation of the multiobjective integer linear programming (MOILP) model of the problem under consideration is given below.

$$
\text { Minimize }\left[L_{1}, \ldots, L_{m}, A_{1}, A_{2}, A_{3}\right]
$$

subject to

$$
\sum_{i \in I} y_{i j}=1, \quad \forall j \in J
$$




$$
l_{i} \leq \sum_{j \in J} y_{i j} h_{j} \leq u_{i}, \forall i \in I
$$

Error! Objects cannot be created from editing field codes., $\forall t \in T$

$$
\begin{aligned}
& \sum_{j \in J} x_{i j t} \leq d_{t}, \forall i \in I, t \in T \\
& \sum_{t \in T} x_{i j t} \leq A y_{i j}, \forall i \in I, j \in J \\
& \sum_{t \in T} x_{i j t} \geq y_{i j}, \forall i \in I, j \in J \\
& \sum_{i \in I} x_{i j t} \leq B z_{j t}, \forall j \in J, t \in T \\
& \sum_{i \in I} x_{i j t} \geq z_{j, t}, \forall j \in J, t \in T \\
& x_{i j t}, y_{i j}, z_{j t} \in\{0,1\} \forall i \in I, j \in J, t \in T
\end{aligned}
$$

The constraint sets (6) and (7) ensure that each course must be assigned to only one instructor and the weekly load of each instructor must be between his lower and upper limits respectively. The constraint set (8) guarantees that at most $c_{t}$ parallel courses are given on time slot $t$. The constraint set (9) guarantees that the number of time slot $t$ assigned to each instructor $i$ does not exceed $d_{t}$. If any instructor-course pair $(i, j)$ is assigned to some time slot, constraint set $(10)$ forces $y_{i j}=1$. If the pair $(i, j)$ is not assigned to any time slot, then the left-hand sides of the corresponding two inequalities in constraint sets (10) and (11) are zero, and hence, the constraint set (11) forces $y_{i j}=0$. Similarly, if any instructor is assigned to some course-time slot pair $(j, t)$, the constraint set (12) forces $z_{j t}=1$. If no instructor is assigned to the pair $(j, t)$, then the left-hand sides of the corresponding two inequalities in the constraint sets (12) and (13) are zero, and hence, the constraint set (13) forces $z_{j t}=0$. 
Any optimum solution to the MOILP model answers the question of which instructors have to be assigned to which courses at which time slots in order to simultaneously maximize the satisfaction levels of instructors and administration. Such a solution also answers the other questions of which instructors have to be assigned to which courses and which courses have to be assigned to which time slots separately. Although all these assignments are concordantly formulated in the model, they express preferences of conflicting participants. Because of the conflicts between these assignments, any optimum solution not only realizes the assignment with "maximum" preferences (in the sense of Pareto efficiency), but also demonstrates the degrees of satisfaction of the participants, or the weights of importance for the objectives. Many MCDM methods require the use of weights or degrees of importance of objectives. The studies have brought forth the question: How to establish a weight of importance or priority for the objectives? Here we use both Analytic Hierarchy Process (AHP) and the Analytic Network Process (ANP) to obtain these weights. The AHP and ANP are the most effective methods for determining the weights of importance of the different criteria. These methods can be characterized as multi-criteria decision techniques that can combine qualitative and quantitative factors in the overall evaluation of alternatives.

\section{An application - a demonstration example}

We consider a particular problem with 6 instructors, 10 courses and 5 time slots. The preference levels $a_{i j}$, bjt and $p_{i j t}$ are shown in tables 1, 2 and 3-1, 3-2.., 3-6 respectively. For other characteristics of this particular problem see section 4.1. 
Table 1. $a_{i j}$-the administration preferences on giving course $j$ by instructor $i$

\begin{tabular}{|c|c||c|c|c|c|c|c|c|c|c|}
\hline $\boldsymbol{i} \backslash \boldsymbol{j}$ & $\mathbf{1}$ & $\mathbf{2}$ & $\mathbf{3}$ & $\mathbf{4}$ & $\mathbf{5}$ & $\mathbf{6}$ & $\mathbf{7}$ & $\mathbf{8}$ & $\mathbf{9}$ & $\mathbf{1 0}$ \\
\hline $\mathbf{1}$ & 1 & 2 & 3 & 4 & 8 & 9 & 9 & 9 & 9 & 9 \\
\hline $\mathbf{2}$ & 8 & 8 & 8 & 9 & 9 & 1 & 2 & 9 & 9 & 9 \\
\hline $\mathbf{3}$ & 9 & 9 & 9 & 9 & 9 & 8 & 1 & 2 & 3 & 4 \\
\hline $\mathbf{4}$ & 1 & 2 & 3 & 9 & 9 & 9 & 9 & 9 & 9 & 9 \\
\hline $\mathbf{5}$ & 9 & 9 & 9 & 9 & 9 & 9 & 9 & 1 & 2 & 3 \\
\hline $\mathbf{6}$ & 9 & 9 & 9 & 9 & 9 & 9 & 9 & 3 & 2 & 1 \\
\hline
\end{tabular}

Table 2. bjt - the administration preference levels on giving the course $j$ on time $t$.

\begin{tabular}{|c|c|c|c|c|c|}
\hline $\boldsymbol{j} \backslash \boldsymbol{t}$ & $\mathbf{1}$ & $\mathbf{2}$ & $\mathbf{3}$ & $\mathbf{4}$ & $\mathbf{5}$ \\
\hline $\mathbf{1}$ & 1 & 3 & 5 & 6 & 9 \\
\hline $\mathbf{2}$ & 1 & 2 & 3 & 6 & 9 \\
\hline $\mathbf{3}$ & 1 & 2 & 3 & 4 & 9 \\
\hline $\mathbf{4}$ & 2 & 1 & 3 & 6 & 9 \\
\hline $\mathbf{5}$ & 3 & 1 & 2 & 5 & 9 \\
\hline $\mathbf{6}$ & 3 & 2 & 1 & 4 & 9 \\
\hline $\mathbf{7}$ & 4 & 3 & 1 & 2 & 6 \\
\hline $\mathbf{8}$ & 6 & 5 & 4 & 1 & 2 \\
\hline $\mathbf{9}$ & 7 & 6 & 5 & 2 & 1 \\
\hline $\mathbf{1 0}$ & 9 & 7 & 6 & 2 & 1 \\
\hline
\end{tabular}


Table 3-1. $p_{1 j t}$ - preference levels for $1^{\text {st }}$ instructor on course-time slot assignments.

\begin{tabular}{|c|c|c|c|c|c|}
\hline $\boldsymbol{j} \backslash \boldsymbol{t}$ & $\mathbf{1}$ & $\mathbf{2}$ & $\mathbf{3}$ & $\mathbf{4}$ & $\mathbf{5}$ \\
\hline $\mathbf{1}$ & 1 & 2 & 3 & 7 & 9 \\
\hline \hline $\mathbf{2}$ & 2 & 1 & 3 & 7 & 9 \\
\hline \hline $\mathbf{3}$ & 3 & 2 & 1 & 6 & 9 \\
\hline \hline $\mathbf{4}$ & 9 & 6 & 2 & 1 & 3 \\
\hline $\mathbf{5}$ & 9 & 6 & 1 & 2 & 7 \\
\hline $\mathbf{6}$ & 9 & 9 & 9 & 9 & 9 \\
\hline $\mathbf{7}$ & 9 & 9 & 9 & 9 & 9 \\
\hline $\mathbf{8}$ & 9 & 9 & 9 & 9 & 9 \\
\hline $\mathbf{9}$ & 9 & 9 & 9 & 9 & 9 \\
\hline \hline $\mathbf{1 0}$ & 9 & 9 & 9 & 9 & 9 \\
\hline
\end{tabular}

Table 3-2. $\quad p_{2 j t}$ - preference levels for $2^{\text {nd }}$ instructor on course-time slot assignments.

\begin{tabular}{|c|c|c|c|c|c|}
\hline $\boldsymbol{j} \backslash \boldsymbol{t}$ & $\mathbf{1}$ & $\mathbf{2}$ & $\mathbf{3}$ & $\mathbf{4}$ & $\mathbf{5}$ \\
\hline $\mathbf{1}$ & 1 & 2 & 3 & 7 & 9 \\
\hline $\mathbf{2}$ & 2 & 1 & 3 & 7 & 9 \\
\hline $\mathbf{3}$ & 3 & 2 & 1 & 6 & 9 \\
\hline $\mathbf{4}$ & 9 & 9 & 9 & 9 & 9 \\
\hline $\mathbf{5}$ & 9 & 9 & 9 & 9 & 9 \\
\hline $\mathbf{6}$ & 4 & 2 & 1 & 3 & 6 \\
\hline $\mathbf{7}$ & 6 & 3 & 1 & 2 & 5 \\
\hline $\mathbf{8}$ & 9 & 9 & 9 & 9 & 9 \\
\hline \hline $\mathbf{9}$ & 9 & 9 & 9 & 9 & 9 \\
\hline \hline $\mathbf{1 0}$ & 9 & 9 & 9 & 9 & 9 \\
\hline
\end{tabular}


Table 3-3. $\quad p_{3 j t}$ - preference levels for $3^{\text {rd }}$ instructor on course-time slot assignments.

\begin{tabular}{|c|c|c|c|c|c|}
\hline $\boldsymbol{j} \backslash \boldsymbol{t}$ & $\mathbf{1}$ & $\mathbf{3}$ & $\mathbf{3}$ & $\mathbf{5}$ \\
\hline $\mathbf{1}$ & 9 & 9 & 9 & 9 & 9 \\
\hline $\mathbf{2}$ & 9 & 9 & 9 & 9 & 9 \\
\hline $\mathbf{3}$ & 9 & 9 & 9 & 9 & 9 \\
\hline $\mathbf{4}$ & 9 & 9 & 9 & 9 & 9 \\
\hline $\mathbf{5}$ & 9 & 9 & 9 & 9 & 9 \\
\hline $\mathbf{6}$ & 3 & 2 & 1 & 4 & 6 \\
\hline $\mathbf{7}$ & 3 & 1 & 2 & 5 & 7 \\
\hline $\mathbf{8}$ & 9 & 5 & 3 & 1 & 2 \\
\hline $\mathbf{9}$ & 9 & 7 & 3 & 2 & 1 \\
\hline $\mathbf{1 0}$ & 9 & 6 & 2 & 3 & 1 \\
\hline
\end{tabular}

Table 3-4. $\quad p_{4 j t}$ - preference levels for $4^{\text {th }}$ instructor on course-time slot assignments.

\begin{tabular}{|c|c|c|c|c|c|}
\hline $\boldsymbol{j} \backslash \boldsymbol{t}$ & $\mathbf{1}$ & $\mathbf{2}$ & $\mathbf{3}$ & $\mathbf{4}$ & $\mathbf{5}$ \\
\hline $\mathbf{1}$ & 1 & 2 & 3 & 5 & 7 \\
\hline $\mathbf{2}$ & 2 & 1 & 4 & 6 & 9 \\
\hline $\mathbf{3}$ & 1 & 2 & 3 & 7 & 9 \\
\hline \hline $\mathbf{4}$ & 9 & 9 & 9 & 9 & 9 \\
\hline $\mathbf{5}$ & 9 & 9 & 9 & 9 & 9 \\
\hline $\mathbf{6}$ & 9 & 9 & 9 & 9 & 9 \\
\hline $\mathbf{7}$ & 9 & 9 & 9 & 9 & 9 \\
\hline $\mathbf{8}$ & 9 & 9 & 9 & 9 & 9 \\
\hline $\mathbf{9}$ & 9 & 9 & 9 & 9 & 9 \\
\hline
\end{tabular}




\begin{tabular}{|l|l|l|l|l|l|}
\hline $\mathbf{1 0}$ & 9 & 9 & 9 & 9 & 9 \\
\hline
\end{tabular}

Table 3-5. $\quad p_{5 j t}$ - preference levels for $5^{\text {th }}$ instructor on course-time slot assignments.

\begin{tabular}{|c|c|c|c|c|c|}
\hline $\boldsymbol{j} \backslash \boldsymbol{t}$ & $\mathbf{1}$ & $\mathbf{2}$ & $\mathbf{3}$ & $\mathbf{4}$ & $\mathbf{5}$ \\
\hline $\mathbf{1}$ & 9 & 9 & 9 & 9 & 9 \\
\hline $\mathbf{2}$ & 9 & 9 & 9 & 9 & 9 \\
\hline $\mathbf{3}$ & 9 & 9 & 9 & 9 & 9 \\
\hline $\mathbf{4}$ & 9 & 9 & 9 & 9 & 9 \\
\hline $\mathbf{5}$ & 9 & 9 & 9 & 9 & 9 \\
\hline $\mathbf{6}$ & 9 & 9 & 9 & 9 & 9 \\
\hline $\mathbf{7}$ & 9 & 9 & 9 & 9 & 9 \\
\hline $\mathbf{8}$ & 9 & 4 & 2 & 1 & 3 \\
\hline $\mathbf{9}$ & 9 & 7 & 1 & 3 & 2 \\
\hline $\mathbf{1 0}$ & 7 & 6 & 3 & 2 & 1 \\
\hline
\end{tabular}

Table 3-6. $\quad p_{6 j t}$ - preference levels for $6^{\text {th }}$ instructor on course-time slot assignments.

\begin{tabular}{|c|c|c|c||c|c|}
\hline $\boldsymbol{j} \backslash \boldsymbol{t}$ & $\mathbf{1}$ & $\mathbf{2}$ & $\mathbf{3}$ & $\mathbf{4}$ & $\mathbf{5}$ \\
\hline $\mathbf{1}$ & 9 & 9 & 9 & 9 & 9 \\
\hline $\mathbf{2}$ & 9 & 9 & 9 & 9 & 9 \\
\hline \hline $\mathbf{3}$ & 9 & 9 & 9 & 9 & 9 \\
\hline \hline $\mathbf{4}$ & 9 & 9 & 9 & 9 & 9 \\
\hline $\mathbf{5}$ & 9 & 9 & 9 & 9 & 9 \\
\hline $\mathbf{6}$ & 9 & 9 & 9 & 9 & 9 \\
\hline $\mathbf{7}$ & 9 & 9 & 9 & 9 & 9 \\
\hline $\mathbf{8}$ & 9 & 7 & 6 & 2 & 1 \\
\hline $\mathbf{9}$ & 9 & 6 & 7 & 1 & 2 \\
\hline
\end{tabular}




\begin{tabular}{|l|l|l|l||l||l|}
\hline $\mathbf{1 0}$ & 7 & 6 & 3 & 1 & 2 \\
\hline
\end{tabular}

\section{The Solution, How?}

Different heuristic approaches have been developed for solving timetabling and related problems, see for example Bloomfield and Mc Sharry (1979), Costa, (1994), Dowsland (1997), Melício et al. (2000) and references therein. Ozdemir and Gasimov (2004) developed an exact solution approach for solving the multiobjective nonlinear facultycourse assignment problem with preferences.

In this paper we examine the exact solution approach for solving the FCT problem constructed in the previous section. Exact solution approaches used for solving such problems generally encounter two main difficulties. The first is has to do with the solution time, which increases exponentially with the number of integer decision variables and the second is the nonconvexity of the problem which again is a result of the existence of integer variables, even though the objective and constraint functions in such models are all linear.

Because of the multiobjective nature of the FCT model, a solution process of this problem has been considered in two stages:

1) Scalarization of the given problem, and

2) Solving the scalarized problem.

As we said before, the objective functions' weights that we need for scalarization have been determined using both the AHP and ANP (see section 4.1). Efficient solutions corresponding to both sets of weights have been calculated and the results compared (see section 4.3).

Because of the nonconvexity of the problem under consideration a special scalarization approach proposed by Gasimov (2001), is implemented (see, section 4.2). 
For solving the scalarized problems GAMS/CPLEX solver was used. Documentation and information about GAMS are available via the World Wide Web at the URL: www.gams.com.

\subsection{Calculation of Objective Function Weights Using Analytic Hierarchy and Network Processes}

AHP and ANP methods developed by Saaty $(2000,2005)$, are the most effective methods for determining the priorities of the different criteria. The solution approach proposed in this study includes objective functions' priorities obtained using AHP and ANP. The six instructors in the system under consideration are grouped as tenured and recent, each consisting of three teachers. Recent instructors have less than five years work experience. The administration requests them to teach more hours (which mainly include basic course applications) than tenured faculty. The nine objectives were divided into three groups with three functions in each: tenured instructor's objective functions; recent instructor's objective functions and objective functions representing the administration's interests. All the objectives were then weighted by their individual and group priorities and then combined, thus obtaining a single satisfaction function for all the people involved.

The AHP and ANP use different ways to derive priorities. The AHP assumes that the criteria (the objectives in our case) are independent and use a hierarchic structure from top to bottom to weigh them. The ANP considers dependence and feedback among the elements to be compared. Here we calculate the weights by using both methods and then compare the results.

\subsubsection{Calculation of AHP weights}

To keep the paper within reasonable length, we will not give computational details instead we will briefly present the judgment matrix and the corresponding priorities as shown in Table 4. This judgment matrix has been filled by the decision maker via Expert Choice software. 
Table 4. The Paired comparison matrix for objectives and the priority vector

\begin{tabular}{|c|c|c|c|c|c|c|c|c|c|c|}
\hline & A1 & A2 & A3 & L1 & L2 & L3 & L4 & L5 & L6 & $\begin{array}{c}\text { Priority } \\
\text { vector }\end{array}$ \\
\hline A1 & 1 & 2 & 2 & 2 & 3 & 3 & 2 & 2 & 3 & 0.219 \\
\hline A2 & $1 / 2$ & 1 & 1 & 2 & 3 & 3 & 3 & 3 & 3 & 0182 \\
\hline A3 & $1 / 2$ & 1 & 1 & 1 & 2 & 2 & 2 & 2 & 2 & 0.119 \\
\hline L1 & $1 / 2$ & $1 / 2$ & 1 & 1 & 2 & 2 & 1 & 1 & 2 & 0.105 \\
\hline L2 & $1 / 3$ & $1 / 3$ & $1 / 2$ & $1 / 2$ & 1 & 1 & 1 & 1 & 1 & 0.054 \\
\hline L3 & $1 / 3$ & $1 / 3$ & $1 / 2$ & $1 / 2$ & 1 & 1 & 1 & 1 & 1 & 0.070 \\
\hline L4 & $1 / 2$ & $1 / 3$ & $1 / 2$ & 1 & 1 & 1 & 1 & 1 & 1 & 0.090 \\
\hline L5 & $1 / 2$ & $1 / 3$ & $1 / 2$ & 1 & 1 & 1 & 1 & 1 & 1 & 0.090 \\
\hline L6 & $1 / 3$ & $1 / 2$ & $1 / 2$ & $1 / 2$ & 1 & 1 & 1 & 1 & 1 & 0.070 \\
\hline
\end{tabular}

\subsubsection{Calculation of ANP weights}

To construct an ANP structure, we need to add the parties that are influenced by the problem stakeholders as well as those that they have an influence on. For our problem, instructors and the administration are the parties that affect and/or are also affected by one another. We list our objectives as a separate cluster named alternatives since our purpose is to obtain their relative priorities. The ANP network structure of the problem is shown in Figure 1. For an ANP application, we perform paired comparisons on the elements within the clusters themselves according to their influence on each element in another cluster they are connected to (outer dependence) or on elements in their own cluster (inner dependence). In making comparisons, one must always has a criterion in mind. Comparisons of elements according to which element influences a given element more and how strongly more than another element it is compared with are made with a control criterion in mind. According to Figure 1, recent instructors have an influence on the alternatives, and also on the administration. These elements also influence recent instructors. So there is dependence among them in both ways. When we only consider the recent instructor cluster, the paired comparison questions arise as a result of its connections to other clusters as can be seen in Figure 2. According to this view, the administration has three times more influence than alternatives on recent instructor. 
Similarly, alternatives have two times more influence than recent instructors on recent instructors.

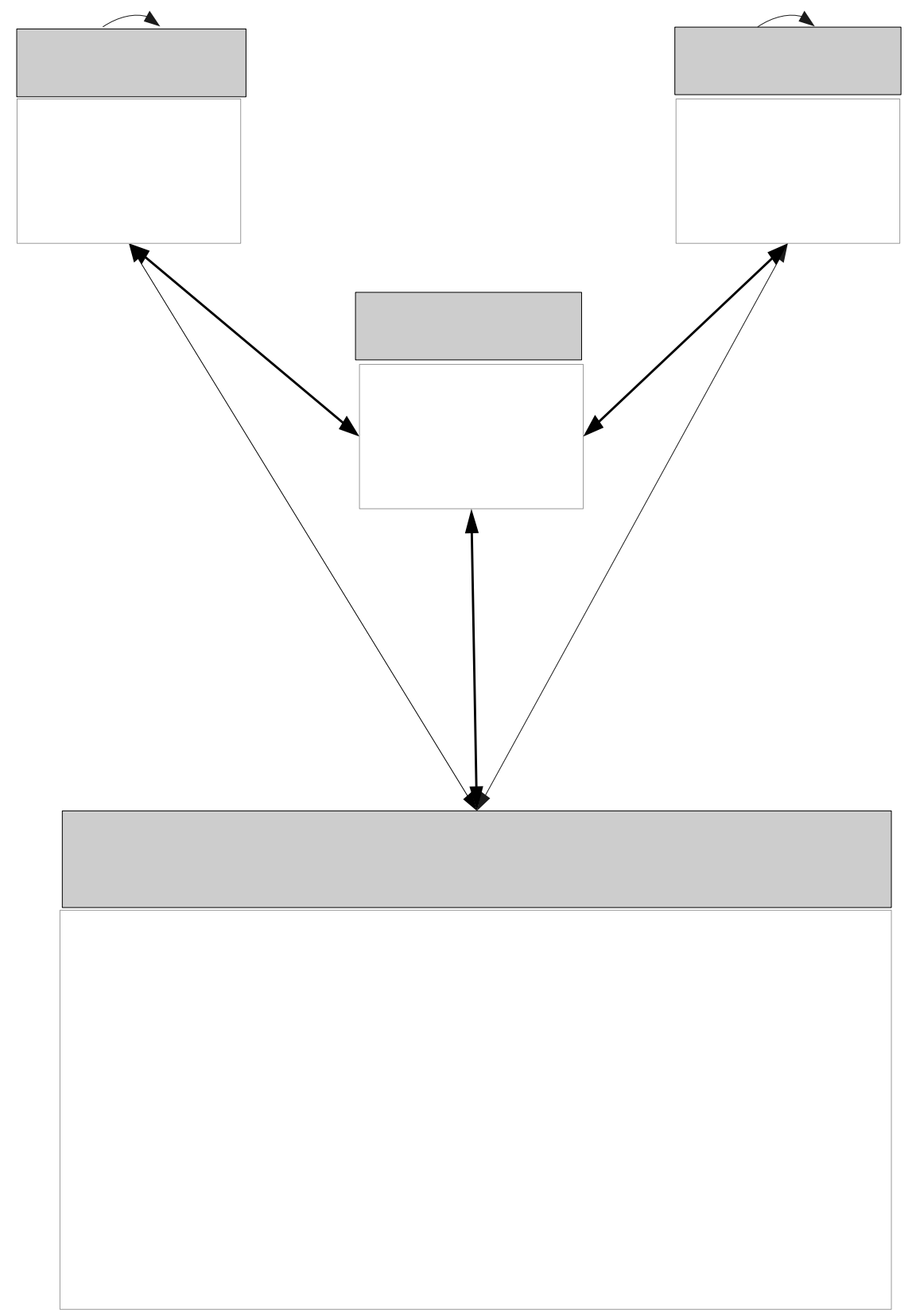

Figure 1. ANP structure of the problem Instructor 1 Instructor 4 


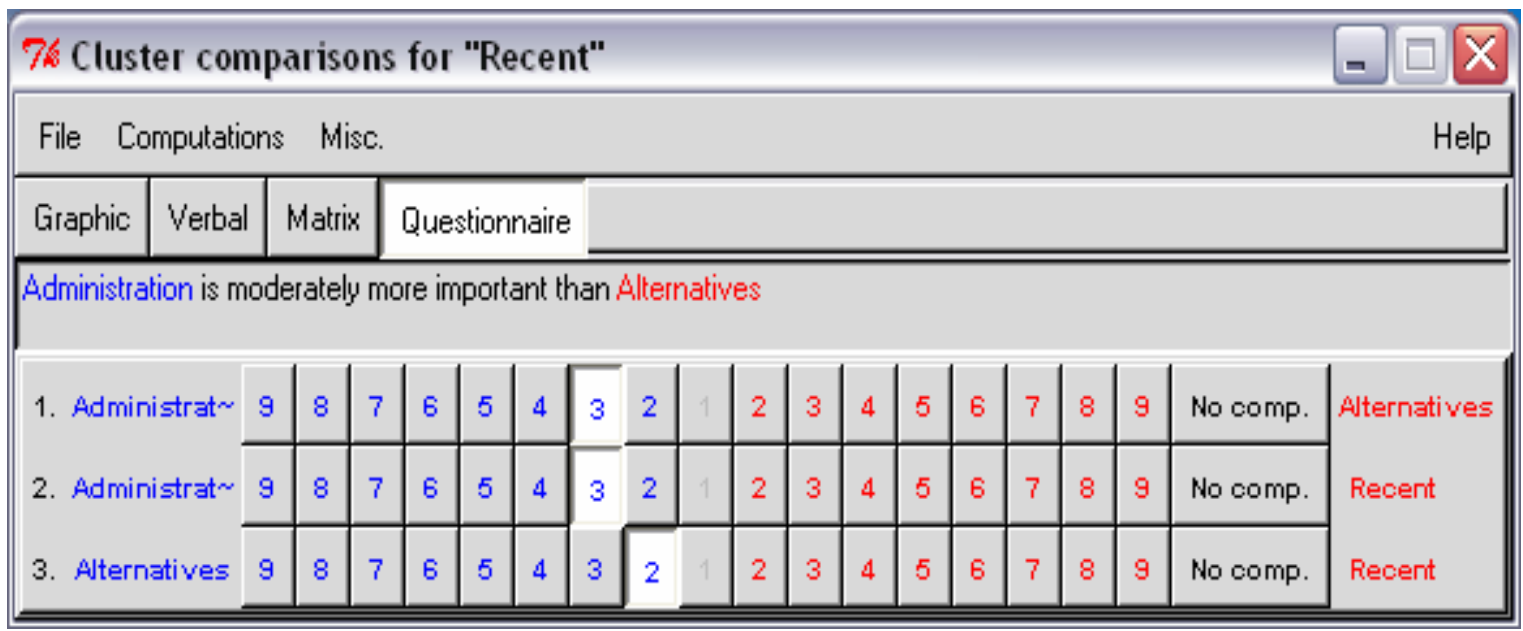

Figure 2. A screen view

Each paired comparison set gives local priorities. By considering the set of previous comparison, the administration has the most influence on recent instructor with priority of 0.593 followed by alternatives with priority 0.249 and recent instructors come last with priority of 0.157 .

Since our objective is to find the overall priority values for each objective, we perform paired comparisons on all clusters as they influence each cluster to which they are connected with respect to the given control criterion. Table 5 gives the objective function weights for the entire ANP model.

Table 5. Overall Outcome

\begin{tabular}{|c|c|c|c|c|c|c|c|c|c|}
\hline Objective & $L_{1}$ & $L_{2}$ & $L_{3}$ & $L_{4}$ & $L_{5}$ & $L_{6}$ & $A_{1}$ & $A_{2}$ & $A_{3}$ \\
\hline Weight $\left(w_{i}\right)$ & 0.087 & 0.046 & 0.065 & 0.074 & 0.087 & 0.045 & 0.184 & 0.251 & 0.157 \\
\hline
\end{tabular}

Table 6 lists both sets of objective function weights obtained by the AHP and ANP. 
Table 6. Objective function weights obtained by AHP and ANP

\begin{tabular}{|c|c|c|}
\hline $\begin{array}{c}\text { Objective } \\
\text { functions }\end{array}$ & ANP weights & AHP weights \\
\hline $\mathbf{A 1}$ & $0.184 \times 1000=184$ & $0.219 \times 1000=219$ \\
\hline $\mathbf{A 2}$ & $0.251 \times 1000=251$ & $0.182 \times 1000=182$ \\
\hline $\mathbf{A 3}$ & $0.157 \times 1000=157$ & $0.119 \times 1000=119$ \\
\hline L1 & $0.087 \times 1000=87$ & $0.105 \times 1000=105$ \\
\hline L2 & $0.046 \times 1000=46$ & $0.054 \times 1000=54$ \\
\hline L3 & $0.065 \times 1000=65$ & $0.070 \times 1000=70$ \\
\hline L4 & $0.074 \times 1000=74$ & $0.090 \times 1000=90$ \\
\hline L5 & $0.087 \times 1000=87$ & $0.090 \times 1000=90$ \\
\hline L6 & $0.045 \times 1000=45$ & $0.070 \times 1000=70$ \\
\hline
\end{tabular}

\subsection{Scalarization}

Scalarization means combining different objectives into a single objective in such a way that repeatedly solving the single objective optimization problem with varying parameters allows us to find all efficient (or properly efficient) solutions of the initial multiobjective problem. Many scalarization methods are known; see Chankong and Haimes (1983), (Luc 1989), Gasimov (2001), Rubinov and Gasimov (2004) and Ehrgott (2005).

We use here the so called conic scalarization approach proposed by Gasimov (2001). Gasimov introduced a class of increasing convex functions to scalarize the multiobjective problem without any assumptions on objectives and constraints of the problem under consideration. This approach is based on supporting the image set of the problem by using cones instead of hyperplanes used in the weighted scalarization. Another advantage of this approach is that it preserves convexity, if the objective functions and constraints of the initial problem are linear or convex. Note that this approach has been successfully applied to a multiobjective 0-1 faculty course assignment problem studied by Ozdemir and Gasimov (2004). Now we briefly present the main scalarization results of Gasimov (2001). 
Let $R_{+}^{9}=\left\{u=\left(u_{1}, \ldots, u_{9}\right) \in R^{9} \mid u_{i} \geq 0, i=1, \ldots, 9\right\}$.

Definition 1 Let $S$ be a nonempty subset of $R^{9}$.

a) An element $s \in S$ is called a Pareto minimal element of the set $S$, written $s \in \min (S)$, if $\left(\{s\}-R_{+}^{9}\right) \cap S=\{s\}$.

b) An element $s \in S$ is called a properly minimal element of $S$ (in the sense of Benson), written $s \in p-\min (S)$, if $s$ is a Pareto minimal element of $S$ and the zero element of $R^{9}$ is a Pareto minimal element of cl cone $\left(S+R_{+}^{9}-\left\{s_{\}}\right)\right.$, where cl denotes the closure of the set and cone $(S):=\{\lambda s \mid \lambda \geq 0$ and $s \in S\}$.

We can formulate our FCT problem in the form:

$$
(\operatorname{MOILP}) \quad \min _{(x, y, z) \in X}\left[F_{1}(x, y, z), \ldots, F_{9}(x, y, z)\right]
$$

where $X$ is the set of binary feasible solutions defined by the constraints (6)-(14), and the functions $F_{1}-F_{6}$ correspond to functions $L_{1}-L_{6}$, and $F_{7}-F_{9}$ to $A_{1}-A_{3}$ respectively (see (5) and (1)-(4) for corresponding definitions).

Let $F(x, y, z)=\left(F_{1}(x, y, z), \ldots, F_{9}(x, y, z)\right)$, and let $F(X)$ be the image of $X$.

Definition2 $(\bar{x}, \bar{y}, \bar{z}) \in X$ is called a Pareto efficient solution of (MOILP) if $F(\bar{x}, \bar{y}, \bar{z}) \in \min (F(X)) ; \quad(\bar{x}, \bar{y}, \bar{z}) \in X$ is called a proper efficient solution of $(M O I L P)$ (in the sense of Benson), if $F(\bar{x}, \bar{y}, \bar{z}) \in p-\min (F(X))$.

Let

$$
W:=\left\{(\alpha, w) \in R \times R^{9} \mid 0 \leq \alpha<\min \left\{w_{1}, \ldots, w_{9}\right\}, w_{1}, \ldots, w_{9}>0\right\} .
$$

Theorem 1 Suppose that for some $(\alpha, w) \in W$ an element $(\bar{x}, \bar{y}, \bar{z}) \in X$ is an optimal solution to the following scalar minimization problem:

$$
\min _{(x, y, z) \in X}\left[\alpha \sum_{i=1}^{9}\left|F_{i}(x, y, z)\right|+\sum_{i=1}^{9} w_{i} F_{i}(x, y, z)\right] .
$$

Then the triple $(\bar{x}, \bar{y}, \bar{z}) \in X$ is a proper efficient solution of (MOILP). 
Theorem 2 Suppose $(\bar{x}, \bar{y}, \bar{z}) \in X$ is a proper efficient solution of (MOILP). Then there exists an element $(\alpha, w) \in W$, such that $(\bar{x}, \bar{y}, \bar{z}) \in X$ is an optimal solution to the following scalar minimization problem:

$$
\min _{(x, y, z) \in X}\left\{\alpha \sum_{i=1}^{9}\left|F_{i}(x, y, z)-F_{i}(\bar{x}, \bar{y}, \bar{z})\right|+\sum_{i=1}^{9} w_{i}\left[F_{i}(x, y, z)-F_{i}(\bar{x}, \bar{y}, \bar{z})\right]\right\} .
$$

Theorem 1 asserts that any solution of the (scalar) problem (16) is an efficient solution of the problem $(M O I L P)$. On the other hand, Theorem 2 claims that every efficient solution $(\bar{x}, \bar{y}, \bar{z})$ of $($ MOILP $)$ can be calculated by solving a scalar problem of the form (17) for some pair $(\alpha, w) \in W$. Thus, these theorems assert that the problem (MOILP) can be scalarized in the form (16) and/or (17), and all efficient solutions of $(P)$ can be calculated by solving scalar problems of these forms. Regarding the solutions of problem (16), note that in the case where the signs of the objective functions remain unchanged in the set of feasible solutions, then the absolute values in (16) are not essential in the whole expression and it reduces to an expression representing the weighted scalarization. This situation can be overcome as follows. It is easy and well-known to show that the following two problems have the same set of efficient solutions:

$$
\begin{array}{r}
\min _{(x, y, z) \in X}\left[F_{1}(x, y, z), \ldots, F_{9}(x, y, z)\right], \text { and } \\
\min _{(x, y, z) \in X}\left[F_{1}(x, y, z)-B_{1}, \ldots, F_{9}(x, y, z)-B_{9}\right],
\end{array}
$$

where $B_{1}, \ldots, B_{9}$ are arbitrary fixed numbers. By choosing these numbers in the interior of ranges of $F_{1}, \ldots, F_{9}$ respectively, we can replace the objective functions by new ones for which the conic scalarization approach will work more effectively. When the multiobjective problem under consideration is nonconvex, by taking different values for the parameter $\alpha$ and the numbers $B_{1}, \ldots, B_{9}$, different efficient solutions corresponding to the same set of weights $w_{1}, \ldots, w_{9}$, can be obtained by solving the scalarized version of the problem (18) of the form:

$$
\min _{(x, y, z) \in X}\left\{\alpha \sum_{i=1}^{9}\left|F_{i}(x, y, z)-B_{i}\right|+\sum_{i=1}^{9} w_{i}\left[F_{i}(x, y, z)-B_{i}\right]\right\} .
$$




\subsection{Numerical results}

The demonstration example constructed above is solved for different values of the parameter $\alpha$ and the same set of weights obtained by AHP and ANP. The corresponding objective functions' values along with the values of $\alpha$, are presented in Tables 7 and 8 respectively.

Table 7. Objective functions' values obtained for AHP weights and different $\alpha$ values

\begin{tabular}{|c|c|c|c|c|c|}
\hline $\begin{array}{l}\text { Objective } \\
\text { functions }\end{array}$ & $\boldsymbol{\alpha}=\mathbf{0}$ & $\boldsymbol{\alpha}=\mathbf{1 0}$ & $\boldsymbol{\alpha}=\mathbf{3 0}$ & $\boldsymbol{\alpha}=\mathbf{4 0}$ & $\boldsymbol{\alpha}=\mathbf{4 5}$ \\
\hline \hline $\mathbf{A 1}$ & 28 & 28 & 28 & 28 & 28 \\
\hline $\mathbf{A 2}$ & 15 & 15 & 15 & 15 & 15 \\
\hline $\mathbf{A 3}$ & 38 & 38 & 38 & 38 & 38 \\
\hline $\mathbf{L 1}$ & 9 & 9 & 9 & 9 & 9 \\
\hline $\mathbf{L 2}$ & 8 & 8 & 8 & 8 & 8 \\
\hline $\mathbf{L 3}$ & 4 & 4 & 4 & 4 & 4 \\
\hline \hline $\mathbf{L 4}$ & 6 & 6 & 6 & 6 & 6 \\
\hline $\mathbf{L 5}$ & 4 & 4 & 4 & 4 & 4 \\
\hline \hline L6 & 4 & 4 & 4 & 4 & 4 \\
\hline
\end{tabular}

As can be seen from Tables 7 and 8, no different solutions have been obtained for the AHP weights using simple weighted and conic scalarization methods. In these tables the column $\alpha=0$ corresponds to the weighted scalarization case, see (19). On the other hand, weighted and conic scalarization methods gave two different Pareto efficient solutions for the ANP weights. As it follows from the explanations on the conic scalarization approach presented in Section 4.2, this approach can provide many efficient solutions corresponding to the same set of weights for objective functions. By varying the parameters $\alpha$. and $B_{i}$, it is possible to calculate different efficient solutions. It is remarkable that two different solutions have been obtained for the same (ANP) weights in our example, although one of them is the same as the solution obtained for the AHP weights. 
Table 8. Objective functions' values obtained for ANP weights and different $\alpha$ values

\begin{tabular}{|c||c|c||c|c|c|}
\hline $\begin{array}{l}\text { Objective } \\
\text { functions }\end{array}$ & $\boldsymbol{\alpha}=\mathbf{0}$ & $\boldsymbol{\alpha}=\mathbf{1 0}$ & $\boldsymbol{\alpha}=\mathbf{3 0}$ & $\boldsymbol{\alpha}=\mathbf{4 0}$ & $\boldsymbol{\alpha}=\mathbf{4 5}$ \\
\hline $\mathbf{A 1}$ & 28 & 28 & 28 & 28 & 28 \\
\hline $\mathbf{A 2}$ & 13 & 13 & 15 & 15 & 15 \\
\hline \hline A3 & 38 & 38 & 38 & 38 & 38 \\
\hline L1 & 9 & 9 & 9 & 9 & 9 \\
\hline L2 & 8 & 8 & 8 & 8 & 8 \\
\hline L3 & 4 & 4 & 4 & 4 & 4 \\
\hline L4 & 4 & 4 & 6 & 6 & 6 \\
\hline L5 & 8 & 8 & 4 & 4 & 4 \\
\hline L6 & 9 & 4 & 4 & 4 \\
\hline
\end{tabular}

\section{Conclusions}

In this study a new multiobjective model for faculty course time slot assignment problem has been developed. This model considers faculty-course-time slot assignments in a single stage with paired faculty-course and course-time slots assignments. The significance of our model is that it considers all the instructors' and administration's preferences on faculty-course-time slot assignments and faculty-course, course-time slot assignments respectively. Considering the pedagogical aspects of such assignments is an important contribution to the performance of an educational system.

By using an exact solution approach Pareto efficient solutions have been calculated for a demonstration example that is constructed. The solution approach implemented here uses priorities obtained by the AHP and ANP. These priorities are used in a conic scalarization method for combining different and conflicting objectives and the scalarized problems are solved by applying GAMS/CPLEX solver. 
It is remarkable that two different sets of solutions have been obtained for the same set of weights (calculated by the ANP), which is an important feature provided by the conic scalarization method.

This study can be considered as an important stage in the classical course scheduling problem. It is very important that the term time slots used in this paper relates not to a specific time block of the week, but to a specific partitioning of a working day, considering the pedagogical aspects. By using the outcomes of this problem, the more general timetabling problems in educational institutions can be solved more effectively.

\section{References}

Asratian A.S., de Werra D., A Generalized Class Teacher Model for Some Timetabling Problems, European Journal of Operational Research, 143, 531-542, 2002.

Badri, M.A., A Two Stage Multiobjective Scheduling Model for Faculty-Course-Time Assignments, European Journal of Operational Research, 94, 1,16-28, 1996.

Bardadym, V.A., Computer-Aided School and University Timetabling: The New Wave, in Burke, E.K., Ross, P. (eds), Practice and Theory of Automated Timetabling, v. 1153, Lecture Notes in Computer Science, pp. 22-45. Springer-Verlag, Berlin, 1996.

Bloomfield, S.D., McSharry, M.M., Preferential course Scheduling, Interfaces, 9, 4, 24$31,1979$.

Chankong, V., Haimes, Y.Y., Multiobjective Decision Making : Theory and Methodology Series, North-Holland Series in System Science and Engineering, Volume 8, 1983.

Costa, D., A tabu search algorithm for computing an operational timetable, European Journal of Operational Research, v. 76, pp. 98-110, 1994. 
Cooper, T.B., Kingston, J.H., The Complexity of Timetable Construction Problems, in Burke, E.K., Ross, P. (eds), Practice and Theory of Automated Timetabling, Lecture Notes in Computer Science, v. 1153, pp. 283-295. Springer-Verlag, Berlin, 1996.

Daskalaki, S., Birbas, T., Housos, E., An Integer Programming Formulation for a Case Study in University Timetabling, European Journal of Operations Research, 153, 1, 117135, 2004.

Daskalaki, S., Birbas, T., Efficient Solutions for a University Timetabling Problem through Integer Programming, European Journal of Operations Research, 160, 1, 106$120,2005$.

Dowsland, K.A., Off-the-peg or made-to measure? Timetabling and Scheduling with SA and TS, in Proceedings of the Second International Conference on the Practice and Theory of Automated Timetabling, pp. 7-26, 1997.

Ehrgott, M., Multicriteria Optimization, Springer Berlin Heidelberg 2005.

Even, S., Itai, A., Shamir, A., On the complexity of timetabling and multicommodity flow problems, SIAM Journal of Computation, v. 5, pp. 691-703, 1976.

Gasimov, R.N., Characterization of the Benson Proper Efficiency and Scalarization in Nonconvex Vector Optimization, in Köksalan, M. and Zionts, S. eds., Multiple Criteria Decision Making in the New Millennium, Lecture Notes in Economics and Mathematical Systems, 507, 189-198, 2001.

Hertz A., Robert, V., Constructing a Course Schedule by Solving a Series of Assignment Type Problems, European Journal of Operation Research, 108, 585-603, 1998.

Kara, I, Ozdemir, M.S., Minmax Approaches to Faculty-Course Assignment Problem, Proceeding of the $2^{\text {nd }}$ International Conference on the Practice and Theory of Automated Timetabling, Toronto, Canada 167-181, 1997. 
Luc, D.T., Theory of vector Optimization, Lecture Notes in Economics and Mathematical Systems, Springer Verlag, Berlin, Germany, Vol. 319, 1989.

Melício, F., Caldeira, P., Rosa, A., Solving Timetabling Problem with Simulated Annealing, Filipe, J. (ed.), Kluwer Academic Press, pp.171-178, 2000.

Ozdemir, M.S. and Gasimov, R. N., The Analytic Hierarchy Process and Multiobjective 0-1 Faculty Course Assignment Problem, European Journal of Operational Research, 157/2, 398-408, 2004.

Rubinov, A.M. and Gasimov, R.N., Scalarization and Nonlinear Scalar Duality for Vector Optimization with Preferences that are not Necessarily a Pre-order Relation, Journal of Global Optimization, 29, 455-477, 2004.

Saaty, T.L., Fundamentals of Decision Making and Priority Theory with the Analytic Hierarchy Process, RWS Publications, Pittsburgh, PA, 2000.

Saaty, T.L., Theory and Applications of the Analytic Network Process: Decisions Making with Benefits, Opportunities, Costs and Risks. RWS Publications, Pittsburgh, PA, 2005.

Schaefer, A., A survey of automated timetabling, Artificial Intelligence Review, v. 13, pp. 87-127, 1999.

de Werra, D. An introduction to timetabling, European Journal of Operations Research, 19, 2, 151-162, 1985. 